University of Nebraska - Lincoln

DigitalCommons@University of Nebraska - Lincoln

\title{
Layered Clustering Communication Protocol for Wireless Sensor Networks
}

\author{
Yong Wang \\ University of Nebraska-Lincoln, ywang@cse.unl.edu \\ Byrav Ramamurthy \\ University of Nebraska-Lincoln, bramamurthy2@unl.edu
}

Follow this and additional works at: https://digitalcommons.unl.edu/cseconfwork

Part of the Computer Sciences Commons

Wang, Yong and Ramamurthy, Byrav, "Layered Clustering Communication Protocol for Wireless Sensor Networks" (2007). CSE Conference and Workshop Papers. 102.

https://digitalcommons.unl.edu/cseconfwork/102

This Article is brought to you for free and open access by the Computer Science and Engineering, Department of at DigitalCommons@University of Nebraska - Lincoln. It has been accepted for inclusion in CSE Conference and Workshop Papers by an authorized administrator of DigitalCommons@University of Nebraska - Lincoln. 


\title{
Layered Clustering Communication Protocol for Wireless Sensor Networks
}

\author{
Yong Wang and Byrav Ramamurthy \\ Department of Computer Science and Engineering \\ University of Nebraska-Lincoln \\ Lincoln, NE 68588-0115 U.S.A \\ \{ywang, byrav\}@cse.unl.edu
}

\begin{abstract}
In this paper, we propose a Layered Clustering Hierarchy (LCH) communication protocol for Wireless Sensor Networks (WSNs). The design of LCH has two goals: scalability and energy-efficiency. In $\mathrm{LCH}$, the sensor nodes are organized as a layered clustering structure. Each layer runs a distributed clustering protocol. By randomizing the rotation of cluster heads in each layer, the energy load is distributed evenly across sensors in the network. Our simulations show that LCH is effective in densely deployed sensor networks. On average, $70 \%$ of live sensor nodes are involved directly in the clustering communication hierarchy. Moreover, the simulations also show that the energy load and dead nodes are distributed evenly over the network. As studies prove that the performance of LCH depends mainly on the distributed clustering protocol, the location of cluster heads and cluster size are two critical factors in the design of LCH.
\end{abstract}

\section{Introduction}

The advances in wireless communication and electronics have enabled the development of low-cost, low-power, multifunctional sensor nodes. These tiny sensor nodes, consisting of sensing, data processing, and communication components, make it possible to deploy Wireless Sensor Networks (WSNs), which represent a significant improvement over traditional wired sensor networks. WSNs can greatly simplify system design and operation as the environment being monitored does not require the communication or energy infrastructure associated with wired networks.

WSNs are expected to be solutions to many applications, such as detecting and tracking the passage of troops and tanks on a battlefield, monitoring environmental pollutants, measuring traffic flows on roads, and tracking the location of personnel in a building. A WSN is usually composed of hundreds or thousands of sensor nodes. These sensor nodes are usually densely deployed and have the capability to collect data and route data back to a base station (BS). The individual sensor nodes in a WSN are inherently resource constrained: they have limited processing speed, storage capacity, and communication bandwidth.

Routing is one of the main research areas in wireless sensor networks. According to the underlying network structure, the routing protocols are classified into three categories: flat, hierarchical, and location-based [3]. In location-based routing protocols, sensor nodes are addressed by means of their locations through GPS (Global Positioning System) or similar systems. Such a network cannot be densely deployed because of the high cost of sensor nodes. In this paper, we consider only densely deployed static WSNs.

The routing challenges and design issues in WSNs are as follows.

Energy: In most settings, sensor nodes must operate for long periods of time with the available supply of batteries. As sensor nodes could use up the limited supply of energy which would then disable the entire network, energyefficiency is a critical factor in WSNs. Hence the routing protocol in WSNs is required to distribute energy consumption evenly over sensor nodes in order to best maximize network lifetime.

Communication: Sensor nodes usually have limited bandwidth and transmission power, which in turn constrains inter-sensor communications.

Computation: The embedded processors in sensor nodes are generally not as powerful as those in nodes of a wired or ad hoc network. As such, sensor nodes may not be able to run sophisticated protocols.

Scalability: The routing protocol is required to run in a network which may contain hundreds or thousands of sensor nodes.

Many routing protocols have been specifically designed for WSNs to resolve these issues. However, it is difficult to strike a balance between energy-efficiency and scalability, especially for large scale WSNs. For example, flat-based routing protocols usually have good scalability while the energy load is not distributed evenly over sensor nodes. Hierarchical-based routing protocols for WSNs are energyefficient but have scalability problems. Hence, designing a suitable routing protocol for WSNs is still an undertaking worthy of investigation. 
In this paper, we propose a Layered Clustering Hierarchy $(\mathrm{LCH})$ protocol for WSNs. Energy-efficiency and scalability are two basic design goals of the $\mathrm{LCH}$ protocol.

The paper is organized as follows: Section 2 introduces the related work on routing protocols. Section 3 describes $\mathrm{LCH}$ protocol. Simulations and results are shown in Section 4. Section 5 presents the conclusions and future work.

\section{Related Work}

A comprehensive survey on routing techniques in WSNs can be found in [3]. Based on the underlying structure, the authors classify the routing techniques into three categories: flat, hierarchical and location-based. Below, we only describe flat and hierarchical-based routing protocols.

In flat networks, each node plays the same role and sensor nodes collaborate together to perform the sensing task. Due to the large number of sensor nodes, such networks are usually data-centric. The base station (BS) sends queries to certain regions and waits for data from sensors located in the selected regions. SPIN [2] [8] and Directed Diffusion [1] are two of the flat-based routing protocols. Both of them are able to work on multihop WSNs. SPIN achieves energy savings by eliminating the transmission of redundant data throughout the network while Directed Diffusion selects the low delay path to save energy. However, both SPIN and Directed Diffusion face the same issues in that the energy load is not distributed evenly over sensor nodes. The sensor nodes close to the base stations consume more energy than the sensor nodes far away from the base stations.

In a hierarchical architecture, the routing is usually divided in two stages: select cluster heads and routing. By randomized rotation of cluster heads, the energy load can be distributed evenly over sensor nodes. LEACH [7] [9] is a cluster-based protocol. It achieves energy saving in three ways: randomized rotation of cluster heads, sleep mode and data fusion. PEGASIS [6] is another hierarchical-based routing protocol. It avoids cluster formation and uses a near optimal chain to transmit data to the BS. Both of them are energy-efficient. However, they all assume that the sensor nodes are able to communicate with each other directly, a feature which may not be available for many WSNs.

The study in [5] presents a layered clustering communication protocol for IP multicast network. The layered clustering structure can also be used to organize WSNs.

\section{Layered Clustering Hierarchy Protocol}

The LCH protocol is designed with two basic goals in mind: scalability and energy-efficiency. LCH includes the following features:
- the network is organized as layers based on the nodes' hop distance to the base station,

- each layer runs a distributed clustering protocol,

- the routing is a hybrid of flat and clustering-based routing,

- it is scalable and energy-efficient.

In LCH protocol, the nodes organize themselves into layers according to the number of hops each of them take to the base station respectively. Each layer runs a distributed clustering protocol and selects several nodes as clusterheads $(\mathrm{CH})$. All non-cluster-head nodes will then decide which cluster to join. The cluster-head nodes will receive data from all the cluster members and transmit data to their upper layer. Data aggregation and fusion could happen in each node. However, by randomized rotation of cluster heads in each layer, the energy load can be distributed evenly in the network and thus enable $\mathrm{LCH}$ to attain the maximal network lifetime.

The $\mathrm{LCH}$ protocol can be divided into two stages: initialization stage and distributed clustering protocol stage. The initialization stage takes place at the beginning of the protocol when the base station finds the hop distance of each sensor node by flooding. Then, the operation of LCH is divided into rounds. Each round begins with a cluster formation phase when the layered clusters are organized, followed by a data transmission phase where frames of data are transferred from the nodes to the cluster-head and on to the base station.

\subsection{Initialization Stage}

The hop distance can be found by flooding a message from base station to each sensor node (Figure 1). Initially, only sensor nodes within the reach of the base station can receive the flooding message (Figure 1.a). From the responses of 1-hop sensor nodes (Figure 1.b), the base station sends requests to 1-hop sensor nodes to explore the next hop nodes (Figure 1.c). The 1-hop nodes will act as the local base station for the next hop nodes (Figure 1.d). The flooding will continue until there is no response from the next hop sensor nodes. Thus a sensor node may receive several flooding messages with different hop count from which it will select the minimum option as its hop count.

Throughout the process, each node maintains its parent information. After the initialization stage, the reverse paths are built to BS (Figure 1.e).

Based on the hop distance to the base station, the sensor network can be categorized into layers. Each layer consists of same hop count sensor nodes. We use layer $i$ to represent the nodes with a hop count of $i$. Figure 1.f shows a layered structure after the initialization stage. 


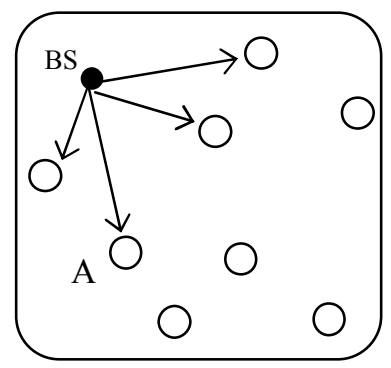

(a)

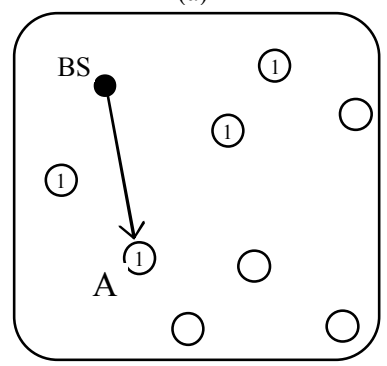

(c)

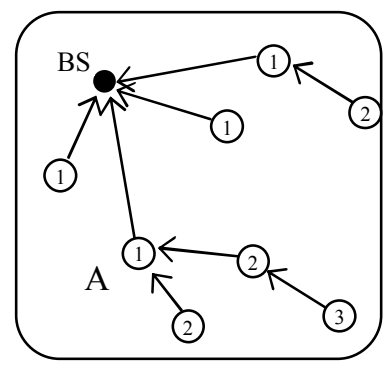

(e)

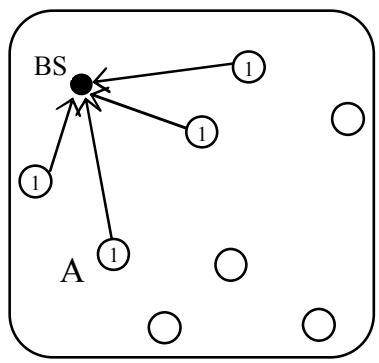

(b)

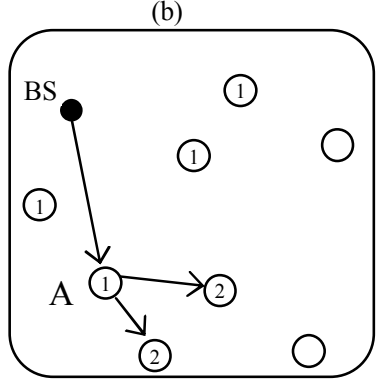

(d)

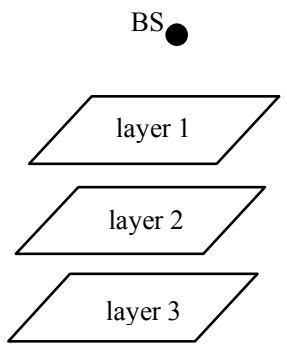

(f)
Figure 1. Initialization stage. BS starts to broadcast message to 1-hop nodes (a); 1-hop nodes send response to BS (2); BS requests A to flood the message (c); A floods message to its neighbors (d); Reverse paths to BS (e); A layered structure (f). The number of hops is shown in the circle.

In the cluster formation phase, sensors select themselves to be cluster-heads with a certain probability in each layer. This probability is chosen such that the expected number of cluster-head nodes is $k_{i}$.

$$
P_{j}(t)=\left\{\begin{array}{cl}
\frac{k_{i}}{N_{i}-k_{i} *\left(r \bmod \frac{N_{i}}{k_{i}}\right)} & : C_{j}(t)=1 \\
0 & : C_{j}(t)=0
\end{array}\right.
$$

where $N_{i}$ is the total node numbers in layer $i, \quad \mathrm{r}$ is the number of rounds that has passed and $C_{j}(t)=0$ if node $i$ has already been a cluster-head in the most recent $\left(r \bmod \frac{N_{i}}{k_{i}}\right)$ rounds and 1 otherwise [7] [9]. The total number of cluster-heads is $\sum_{\text {layers }} k_{i}$.

Once the nodes have elected themselves to be clusterheads, the cluster-head nodes broadcast an announcement powered at the maximum transmission in order to attract other non-cluster-head nodes. Then, the cluster-heads wait for join request messages.

Each non-cluster-head node determines to which cluster it belongs by randomly choosing the best 3 cluster-heads that require the minimum communication energy and sends a join request to the cluster-head. By randomly choosing the best 3 cluster-heads, LCH aims to decrease size difference among clusters. It is possible that some nodes receive cluster-head announcement from different layers. We do not impose any restriction whereby non-cluster-head nodes can only join the same layer of cluster-heads. Meanwhile, the non-cluster-head nodes also update its parent information to its cluster-head.

Cluster-head nodes can also receive cluster-head announcement which may come from different layers. In such a case, the cluster-head will select its upper layer cluster-head which requires minimum communication energy as its cluster-head and update its parent information to this cluster-head.

After receiving the join request message from noncluster-head nodes, the cluster-head node creates a TDMA schedule and sends it to cluster members. Non-cluster-head nodes update their schedules from cluster-head nodes.

After clustering formation phase, each layer consists of several clusters with a cluster-head in each cluster. The network is organized as a layered clustering hierarchy (Figure 2). Most of the nodes are directly involved in some clusters (either as a cluster-head or a cluster member). It is also possible that there are some nodes which are not involved in any clusters, for example, A, B and C in Figure 2. These nodes may indirectly participate in a cluster if any of their upstream nodes are in a cluster. Figure 3 combines the parent information maintained by each node with the layered clustering hierarchy. Some alternative scenarios are also listed in Figure 3. For example, for those non-cluster sensor nodes A, B, and C, B and C are indirectly involved in some clusters. The clustering formation phase is followed by the data transmission phase.

\subsection{Distributed Clustering Protocol Stage}

At this stage, the sensor nodes in each layer will form clusters. Each layer will select several cluster-heads and non-cluster-head members and then decide which cluster to join. The process is similar to LEACH but it is not identical. In LEACH, there is only one layer and the rotation of cluster heads runs only on that layer. 
By randomized rotation of cluster heads in each layer, LCH aims to distribute energy consumption evenly over sensor nodes. This process is especially important for intermediate sensor nodes because they take more responsibility to forward messages.

The distributed clustering protocol is broken up into rounds and each round can be further divided into cluster formation phase and data transmission phase.

- BS
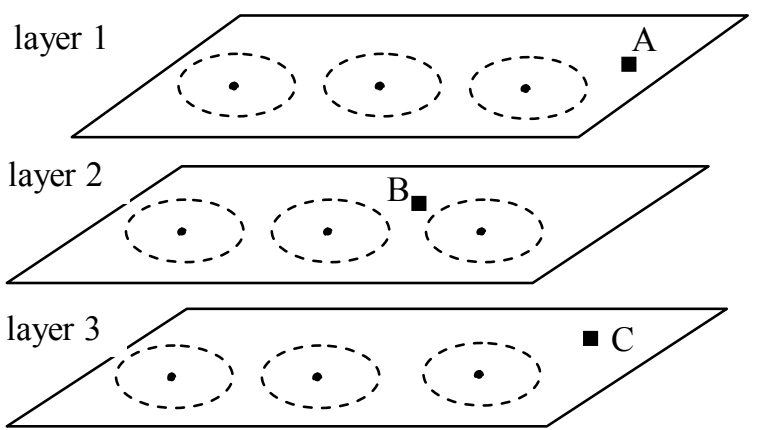

Figure 2. Layered clustering hierarchy. Distributed clustering protocol runs in each layer. Most of nodes are directly involved in the clustering hierarchy. It is possible that there are some nodes which are not in any clusters, for example, A, B and C.

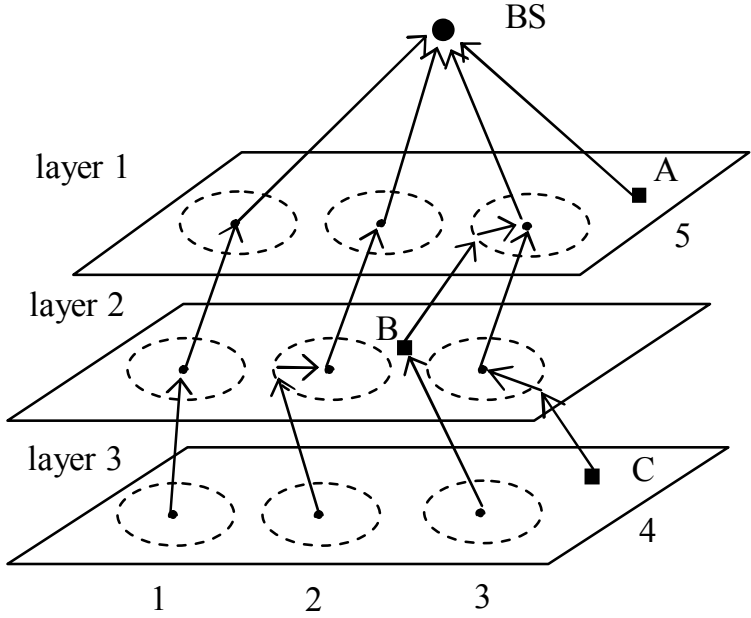

Figure 3. Layered clustering hierarchy with routing. A cluster-head sends data to upper layer cluster-head (1); A cluster-head sends data to a cluster member from the upper layer (2); A cluster-head sends data to a non-cluster node (3); A non-cluster node sends data to its parent which is in a cluster (4). A non-cluster node in layer 1 sends data to BS (5).

\subsection{Data Transmission}

At this stage, each cluster-head will check non-clusterhead nodes by the TDMA schedule. The data transmission is divided into frames. Each non-cluster-head node sends its data to the cluster-head at most once per frame during its allocated transmission slot. In the remaining slots, its radio is turned off (sleep mode) to save energy.

Each cluster-head node receiving data will send the data to its parent. The parent nodes will then forward the message to base station. Data aggregation and fusion may happen as required.

After a time interval, the network will advance into the next round.

\section{Simulations and Results}

The simulation is done on ns2 [15]. The implementation of LCH protocol is based on the module of MIT $\mu$ AMPS LEACH ns Extension [4].

We create a 400-node sensor network. This network is randomly generated and the 400 sensor nodes distributed uniformly in a $200 \mathrm{~m} \times 200 \mathrm{~m}$ area. The power of the sensor radio transmitter is set so that any node within an 80 meter radius is within communication range. The channel capacity is $1 \mathrm{Mbps}$ and the power dissipation is set to $50 \mathrm{~mW}$ for both transmit mode and receive mode. The processing delay for transmitting a message is randomly chosen between 0 and $50 \mu \mathrm{s}$. The number of cluster-heads in each layer is set to 6 . The size of each data item is set to 500 bytes. Each node is initialized with $2 \mathrm{~J}$ of energy. Table 1 summarizes these network characteristics.

Table 1. Simulation Parameters

\begin{tabular}{|l|c|}
\hline Nodes & 400 \\
\hline Network size & $200 \mathrm{~m} \times 200 \mathrm{~m}$ \\
\hline Antenna reach & $80 \mathrm{~m}$ \\
\hline Radio propagation speed & $3 \times 10^{8} \mathrm{~m} / \mathrm{s}$ \\
\hline Processing delay & $50 \mu \mathrm{s}$ \\
\hline Channel capacity & $1 \mathrm{Mbps}$ \\
\hline Transmit cost & $50 \mathrm{~mW}$ \\
\hline Receive cost & $50 \mathrm{~mW}$ \\
\hline Data size & $500 \mathrm{bytes}$ \\
\hline \hline Base station location & $\mathrm{x}=0, \mathrm{y}=200$ \\
\hline
\end{tabular}

Using this network configuration, we ran $\mathrm{LCH}$ and tracked its progress. For each experiment, we ran the protocol 10 times and averaged the data to account for the random processing delay.

Figure 4 shows that the test network is divided into 4 layers after the initial stage. At a time, there are 6 clusterheads in layer 2 . Simulations show that $77 \%$ of cluster size is between 5 and 30 and $6 \%$ of cluster size is greater than 30 nodes (Figure 5 ).

Figure 6 shows the dead nodes in the network. Layer 1, 2 and 3 almost have the same percentage of dead nodes. The test network contains only about 20 nodes in layer 4, thus layer 4 does not follow the trend observed in other 
layers. Figure 6 shows that the dead nodes are distributed evenly over sensor nodes.

Figure 7 shows the network lifetime and nodes involved in clusters. The average network lifetime is $420 \mathrm{~s}$. Following that, $48 \%$ of the nodes are alive but with a high energy consumption of $80 \%$ on average. At that time, $\mathrm{LCH}$ cannot form the clustering hierarchy effectively. During the network lifetime, an average of $70 \%$ of live nodes is directly involved in a clustering hierarchy. This data shows that $\mathrm{LCH}$ functions most effectively in densely deployed sensor networks.

In LCH protocol, the goodput is limited by the number of cluster-heads in the first layer. In the data transmission stage, there is at least one frame in each round. Thus if there are 6 cluster-heads in layer 1 and each layer 1 node is involved in a cluster, the goodput is at least $0.8 \mathrm{kbps}$ when the round time is $30 \mathrm{~s}$.

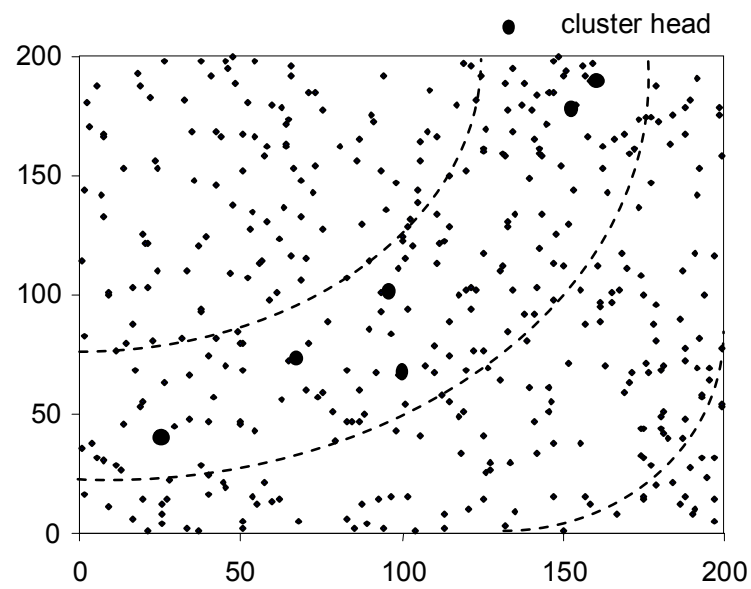

Figure 4. Cluster-heads in layer 2. (Clusters are not shown)

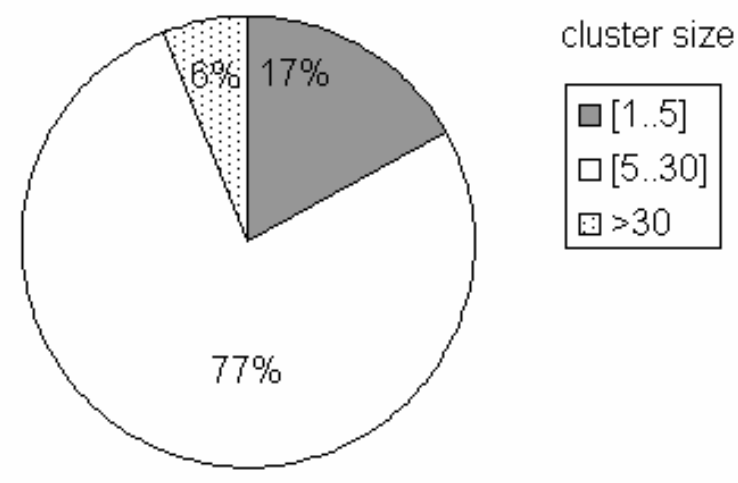

Figure 5. Distribution of cluster size. $77 \%$ of cluster size is between 5 and 30 .

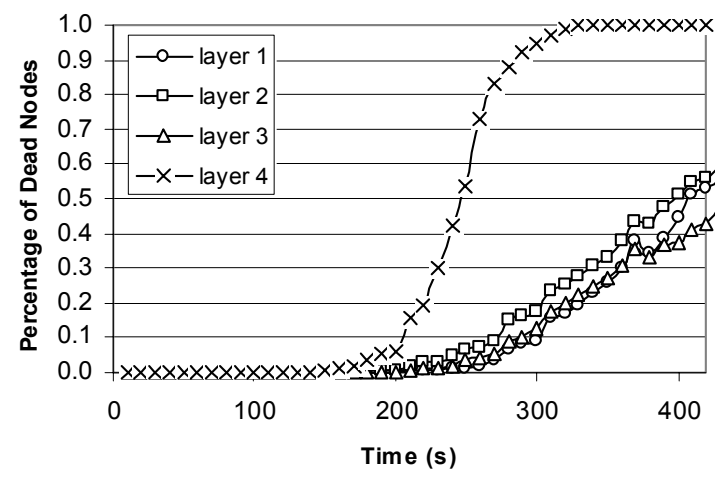

Figure 6. Dead nodes in each layer. Layer 1, 2 and 3 almost have same percentage of dead nodes during the network lifetime.

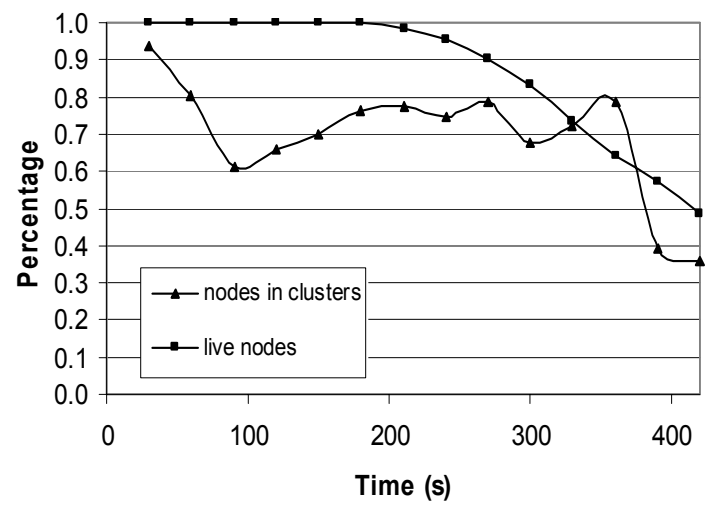

Figure 7. Network lifetime and nodes involved in Clusters. Percentage of live nodes is measured with the total number of nodes in the network. Percentage of nodes in clusters is calculated only on live nodes.

LEACH [7][8] is a special case of the layer communication hierarchy, which includes only one layer. However, LEACH assumes that all the sensor nodes are within reach of each other in the clustering formation phase. LCH has no such limitations.

The performance of $\mathrm{LCH}$ mainly depends on the distributed clustering protocol. Hence the location of cluster-heads and cluster size are two critical factors of the distributed clustering protocol. Several clustering algorithms have been proposed in the context of wireless sensor networks [10] [11] [12]. The proposed clustering algorithms can also be used in the layered clustering communication protocol.

\section{Conclusions and Future Work}

In this paper, we propose a layered clustering hierarchy communication protocol for wireless sensor networks. There are two stages in LCH: the initialization stage, and 
the distributed clustering protocol stage. The distributed clustering protocol can be further divided into cluster formation and data transmission phase.

Our simulations show that LCH is most effective in densely deployed sensor networks. Most of the live sensor nodes $(70 \%)$ can be directly involved in the clustering hierarchy during the network lifetime. Simulations also show that the energy load and the dead nodes are distributed evenly over sensor nodes. Future work includes the evaluation of the performance of the $\mathrm{LCH}$ protocol using different clustering algorithms. Further, a few papers [10]-[14] in the literature have discussed clustering issues in wireless sensor networks and ad hoc networks, the comparison of $\mathrm{LCH}$ protocol with these schemes will also be conducted in the future.

\section{References}

1. C. Intanagonwiwat, R. Govindan, and D. Estrin, "Directed diffusion: a scalable and robust communication paradigm for sensor networks," Proceedings of ACM MobiCom'00, Boston, MA, pp. 56-57, 2000.

2. J. Kulik, W. R. Heinzelman, and H. Balakrishnan, "Negotiation-based protocols for disseminating information in wireless sensor networks," Wireless networks, Volume: 8, pp. 169-185, 2002.

3. J. N. Al-Karaki, and A. E. Kamal, "Routing techniques in wireless sensor networks: a Survey", Wireless Communications, IEEE [see also IEEE Personal Communications], Volume: 11, Issue: 6, pp. 6-28, Dec. 2004.

4. MIT $\mu$-AMPS Project. http://wwwmtl.mit.edu/research/icsystems/uamps/, 1999.

5. S. Banerjee, B. Bhattacharjee, "Scalable Secure Group Communication over IP Multicast", IEEE Journal on
Selected Areas in Communications, Volume: 20, Issue: 8, pp. 1511-1527, October 2002.

6. S. Lindsey, C. Raghavendra, "PEGASIS: Power-Efficient Gathering in Sensor Information Systems", IEEE Aerospace Conference Proceedings, Vol. 3, 9-16 pp.1125-1130, 2002

7. W. Heinzelman, A. Chandrakasan, and H. Balakrishnan, "Energy-Efficient Communication Protocol for Wireless Microsensor networks", in Proc. $33^{\text {rd }}$ Hawaii International Conference on System Sciences (HICS '00), January 2000.

8. W. Heinzelman, J. Kulik, and H. Balakrishnan, "Adaptive Protocols for Information Dissemination in Wireless Sensor Network", in Proceedings of the Fifth annual ACM/IEEE International Conference on Mobile Computing and networking (MobiCom'99), pages 174-185, August 1999.

9. W. Heinzelman, "Application-Specific Protocol Architectures for Wireless Networks", Ph.D. Dissertation, Massachusetts Institute of Technology, June 2000.

10. H. Chan and A. Perrig, "ACE: An emergent algorithm for highly uniform cluster formation," in European Workshop on Wireless Sensor Networks (EWSN 2004), Jan. 2004.

11. S. Bandyopadhyay and E. J. Coyle, "An energy efficient hierarchical clustering algorithm for wireless sensor networks," in Proceedings of IEEE INFOCOM, April 2003.

12. O. Younis and S. Fahmy, "Distributed clustering in ad-hoc sensor networks: A hybrid, energy-efficient approach," in Proceedings of IEEE INFOCOM, March 2004.

13. M. Demirbas, T. Nolte, A. Arora, and Nancy Lynch, "A Hierarchy-based Fault-local Stabilizing Algorithm for Tracking in Sensor Networks," 8th International Conference on Principles of Distributed Systems (OPODIS), France, December 2004.

14. H. Zhang and A. Arora, "GS3: Scalable Self-configuration and Self-healing in Wireless Networks," 21st ACM Symposium on Principles of Distributed Computing (PODC), 2002.

15. The network simulator - ns2. http://www.isi.edu/nsnam/ns/. 